\title{
Expresion del receptor de esteroides y xenobioticos (SXR) y del gen de multirresistencia drogas (MDR1) y de los polimorfismos de las enzimas GSTs, SULTs y CYP en tumores vesicales profundos, analisis de su expresion y correlación con otros factores pronósticos
}

\author{
Rioja Zuazu J*, Bandrés Elizalde E**, Rosell Costa D*, Rincón Mayans A*, Zudaire Bergera J *, \\ Gil Sanz $M^{a} J^{* * *}$, Rioja Sanz LA***, García Foncillas $\mathrm{J}^{* *}$, Berián Polo JM**
}

*Departamento de Urología. Clínica Universitaria. Universidad de Navarra.

**Laboratorio de Farmogenómica. Centro de Investigación Médica Aplicada. Universidad de Navarra. ***Servicio de Urología. Hospital Universitario Miguel Servet. Zaragoza.

Actas Urol Esp. 2007;31(10):1107-1116

RESUMEN

EXPRESION DEL RECEPTOR DE ESTEROIDES Y XENOBIOTICOS (SXR) Y DEL GEN DE MULTIRRESISTENCIA DROGAS (MDR1) Y DE LOS POLIMORFISMOS DE LAS ENZIMAS GSTS, SULTS Y CYP EN TUMORES VESICALES PROFUNDOS, ANALISIS DE SU EXPRESION Y CORRELACIÓN CON OTROS FACTORES PRONÓSTICOS

Introducción: El receptor de esteroides y xenobióticos SXR se ha demostrado su activación por parte de numerosos medicamentos, incluidos potentes inductores del citocromo P450, como la rifampicina y el cotrimazol. La función del SXR es bien conocida, y consiste en regular de manera positiva la trascripción del citocromo P450 3A4 (CYP3A4) y el gen de multirresistencia a drogas (multidrug resistance gene) MDR1, se considera una llave clave en el mecanismo regulador del metabolismo de los xenobióticos encontrándose involucrado en todas las fases de detoxificación

Múltiples enzimas involucradas en el metabolismo y la degradación de hidrocarburos policíclicos aromáticos (PAH) son polimórficas en humanos, incluyendo la glutation S-transferasa (GSTs), N-acetiltransferasa (NATs), sulfotransferas (SULTs) 1A1 y el citocromo p450 (CYP) 1B1.

Objetivos: Los objetivos que nos hemos planteado son los siguientes: 1. Analizar la expresión del factor de trascripción SXR y del MDR1 en vejiga mediante RT-PCR en tiempo real, tanto en vejiga tumoral como vejiga normal. 2. Analizar la relación de los factores clínicos y patológicos con la expresión del SXR y del MDR1. 3. Analizar la expresión de los polimorfismos de CYP1B1, GSTM1 GSTT1 y SULT1A1, y su correlación con distintos factores clínico patológicos y moleculares.

Material y Métodos: De manera prospectiva se calculó un tamaño muestral necesario para este estudio. Se incluyeron 67 pacientes de dos instituciones distintas (Hospital Universitario Miguel Servet (49 HUMS) y Clínica Universitaria de Navarra (18 CUN)), diagnosticados de cáncer vesical infiltrante y tratados mediante cistectomía radical, se le realizó la determinación de la expresión de SXR y MDR1 mediante PCR cuantitativa en tiempo real, así como de los polimorfismos CYP1B1, GSTM1 GSTT1 y SULT1A1 mediante RFLP (restricción de la longitud del fragmento del polimorfismo). Se correlaciona mediante tablas de contingencia la correlación con el resto de los factores pronósticos.

Resultados: La media de seguimiento de los pacientes fue de 23,7 meses, con una mediana de 28,26 meses. De los 67 pacientes estudiados, 31 pacientes $(46,3 \%)$ presentaron progresión de la enfermedad, bien en forma de recidiva local, metástasis a distancia o ambos, con un tiempo medio a recidiva de 12,4 meses, mediana de 10 meses, con un rango de 1,1 mes a 31,9 meses. 36 pacientes $(53,7 \%)$ no presentaron evidencia de progresión de la enfermedad.

El receptor de esteroides y xenobióticos SXR así como el gen de multirresistenia a drogas (Multidrug resistance gene (MDR1)), se expresan en vejiga normal $(0,94 \Delta \mathrm{Ct}$ y $0,94 \Delta \mathrm{Ct})$ y en vejiga tumoral de la pieza de cistectomía ( $1,09 \Delta \mathrm{Ct}$ y $0,45 \Delta \mathrm{Ct})$. Hemos analizado su expresión de manera cuantitativa y de manera cualitativa. La expresión de SXR se correlaciona con la presencia de carcinoma in situ ( $\mathrm{p}=0,024)$, infiltración vasculo-linfática ( $\mathrm{p}=0,05)$ mientras que MDR1 se correlaciona con la presencia de infiltración vasculo linfática $(\mathrm{p}=0,05)$ A su vez ambos la presencia de ambos factores se correlaciona entre ellos ( $\mathrm{p}=0,011)$

Los polimorfismos: CYP1B1, GSTM1, GSTT1 y SULT1A1, se expresan en vejiga pero su expresión no guarda correlación con ningún factor pronóstico

Conclusiones: El SXR y el MDRl se expresan tanto en vejiga normal y tumoral. Y que dicha expresión guarda una correlación con factores pronósticos con influencia en la supervivencia descritas en la literatura.

Palabras clave: Tumores vesical profundos. Biología molecular. Factores pronósticos.

\section{ABSTRACT}

STEROID AND XENOBIOTIC RECEPTOR (SXR), MULTIDRUG RESISTANCE GENE (MDR1) AND GSTs, SULTs AND CYP POLYMORPHISM EXPRESSION IN INVASIVE BLADDER CANCER, ANALYSIS OF THEIR EXPRESSION AND CORRELATION WITH OTHER PROGNOSTIC FACTORS

Introduction: Steroid and Xenobiotic Receptor (SXR) has demonstrated its activation by numerous drugs, including cytochrome P450 potent inducers like rifampicina or cotrimazol. The role of SXR is well known, and lies regulating in a positive manner cytochrome P450 3A4 (CYP3A4) transcription and the multidrug resistance gene (MDR1), it's considered a key in the xenobiotic detoxification mechanism, being involved in all phases of the detoxification process.

Enzymes involved in Policyclic Aromatic hidrocarbures (PAH) metabolism and degradation are polymorphic in humans, including glutation S-transferases (GSTs), N-acetiltransferases (NATs), sulfotransferases (SULTs) 1Al and cytochrome p450 (CYP) 1B1

Objectives: The objectives we've planned are: 1 . Analyze the expression of the transcription factor SXR and MDR1 in bladder by means of RT-PCR real time, both in normal bladder and in tumoral bladder. 2. Analyze the relation between clinical and pathological factors with the expression of SXR and MDR1. 3. Analyze the expression of the polymorphims CYP1B1, GSTM1 GSTT1 and SULT1A1 and their correlation with different clinic-pathological and molecular factors.

Material and Methods: In a prospective way the size of the sample was estimated. In 67 patients from two institutions (Hospital Universitario Miguel Servet (49 HUMS) and Clinica Universitaria de Navarra (18 CUN)), diagnosed of invasive bladder cancer and treated by means of radical cystectomy, were determined the expression of both SXR and MDR1 by means of real time PCR, as well as the polymorphisms CYP1B1, GSTM1 GSTT1 y SULT1A1 by means of RFLP (Restriction fragment length polymorphism). Correlations with other prognostic factors by contingency tables were performed.

Results: Average follow up was 23,7 months with a median of 28,26 months. Of the 67 patients studied, 31 patients $(46,3)$ presented disease progression, in form of local recurrence or in distant metastasis or both. With a average time to progression of 12,4 months and a median of 10 months, with a range of 1,1 month to 31,9 month. 36 patients (53,7\%) did not have any evidence of disease progression during follow up.

The Steroid and Xenobiotic Receptor as well as the Multidrug Resistance Gene (MDR1) are expressed in both normal bladder (0,94 $\Delta \mathrm{Ct}$ y $0,94 \Delta \mathrm{Ct}$ ) and tumoral bladder in the cystectomy specimen(1,09 $\Delta \mathrm{Ct}$ y $0,45 \Delta \mathrm{Ct})$. We've analyzed their expression in a quantitative manner and in a qualitative manner. The expression of SXR correlates with the presence of ca. in situ $(\mathrm{p}=0,024)$, vasculo-lymphatic invasion $(\mathrm{p}=0,05)$ mean while MDR1 correlates with presence of vasculo-lymphatic invasion $(\mathrm{p}=0,05)$ Both factors are correlate between each others $(\mathrm{p}=0,011)$.

Polymorphisms: CYP1B1, GSTM1, GSTT1 and SULT1Al, are expressed in these patients but their expression doesn't correlates with any prognostic factor

Conclusions: Both SXR and MDR1 are expressed in normal bladder as well as in tumoral bladder. And their expression correlates with different prognostic factors with influence in the survival described in the literature

Keywords: Invasive bladder cancer. Molecular biology. Prognostic factors. 
$\mathrm{L}$ a neoplasia vesical es una de las entidades más frecuentemente tratadas por el urólogo ${ }^{1}$. La mayoría, casi el 90\%, de los tumores de urotelio se originan en la vejiga ${ }^{2}$. El cáncer de vejiga supone el $2 \%$ de todos los tumores malignos, la segunda neoplasia urológica, el cuarto cáncer más frecuente en el varón y la décima más frecuente en la mujer ${ }^{3}$. En el año 2005 se han estimado unos 63.210 nuevo casos diagnosticados en Estados Unidos de América (47.010 varones y 16.200 mujeres). En el año 2005 supuso la causa de muerte para 4429 personas en España, 3672 varones y 757 mujeres ${ }^{4}$.

La característica fundamental del cáncer infiltrante de vejiga, es la invasión de la capa muscular, la cuál es un marcador importante de progresión ${ }^{5}$. Se asocia a la presencia de ganglios linfáticos afectados en un 40-50\%, fundamentalmente a nivel pélvico, y con la existencia de metástasis a distancia a lo largo de su evolución en un $50-70 \%$ de los casos.

En el campo de la biología molecular, se han realizado múltiples investigaciones asociadas a esta enfermedad, pero hoy en día continúa sin esclarecerse, de una forma sólida y consistente, ninguna variable o determinante que condicione la práctica clínica diaria de los urólogos y oncólogos.

La modificación de las pautas terapéuticas, así como la combinación de las mismas, y el modo de combinarlas, sigue estando bajo el condicionante de la biología celular, mientras que la biología molecular y sus marcadores aportan información adicional de cara a estudios pronósticos. No habiéndose conseguido consolidar su utilización en la práctica clínica diaria. El objetivo de identificar y de condicionar un tratamiento, más o menos agresivo, en función de la identidad tumoral a nivel molecular, continúa siendo una quimera.

$\mathrm{El}$ receptor de esteroides y xenobióticos $\mathrm{SXR}^{6}$, está codificado por NR1I2(Nuclear Receptor Nomenclatura Homepage ${ }^{7}$, se ha demostrado su activación por parte de numerosos medicamentos, incluidos potentes inductores del citocromo $\mathrm{P} 450$, como la rifampicina y el cotrimazol ${ }^{6,8,9}$. El $\mathrm{SXR}$, a su vez, se ha descrito su presencia a nivel hepático, intestinal, tanto delgado como grueso ${ }^{6}$, 8,9 , y más recientemente a nivel pulmonar $\mathrm{y}$ renal $^{10}$.
La función del SXR es bien conocida, y consiste en regular de manera positiva la trascripción del citocromo P450 3A4 (CYP3A4) y el gen de multirresistencia a drogas (multidrug resistance gene) $\mathrm{MDR} 1^{7,11}$, y se considera una llave clave en el mecanismo regulador del metabolismo de los xenobióticos ${ }^{12-15}$, encontrándose involucrado en todas las fases de detoxificación.

A través de una combinación de abordajes farmacológicos y genéticos, se ha demostrado que el SXR activa la expresión de MDR1 en hepatocitos y células intestinales y que dicha activación, resulta en un mayor aflujo de droga, siendo la primera evidencia de que el SXR regula la coordinación de múltiples vías, tanto del metabolismo como de la excreción de xenobiótios, en distintos tejidos.

La expresión del MDR1 en vejiga se ha demostrado, así como su inducción por agentes quimioterápicos, poniendo en evidencia que la sobreexpresión del MDR1 puede contribuir a la resistencia a agentes antineoplásicos ${ }^{16}$.

La exposición a hidrocarburos policíclicos aromáticos (PAH) y las aminas aromáticas, en su mayoría por el tabaco o por exposiciones laborales, se ha asociado a la carcinogénesis del cáncer de vejiga ${ }^{17}$. Múltiples enzimas involucradas en el metabolismo y la degradación de estas sustancias procancerígenas son polimórficas en humanos, incluyendo la glutation S-transferasa (GSTs), N-acetiltransferasa (NATs), sulfotransferas (SULTs) 1A1 y el citocromo p450 (CYP) 1B1. Se ha hipotetizado que las variaciones genéticas de estas enzimas podrían contribuir a las variaciones interindividuales de la susceptibilidad genética al cáncer de vejiga ${ }^{18}$.

GSTs comprenden una importante familia de enzimas que metabolizan una amplia variedad de xenobióticos, catalizando la conjugación de glutation (GSH) y promoviendo una defensa celular contra los agentes citotóxicos y posibles agentes químicos cancerígenos ${ }^{19}$. GSTM1 detoxifica electrolitos hidrofóbicos derivados del metabolismo de los xenobióticos, incluyendo epóxidos derivados de los $\mathrm{PAH}^{19}$. GSTT1 se ha considerado uno de las enzimas más suicidas de todas las GSTs y exhibe una actividad catalítica distinta comparada con otras GSTs ${ }^{20}$. Las delecciones de GSTM1 y GSTT1, que llevan a la pérdida de la actividad 
enzimática, son relativamente prevalentes en distintas poblaciones. La prevalencia estimada del fenotipo GSTM1 nulo es de alrededor del 50\% y del GSTT1 nulo es de alrededor del 15-25\% en población caucásica y de un $60-80 \%$ en población asiática ${ }^{20}$.

Debido al papel de las enzimas GSTs en el metabolismo de cancerígenos, se han desarrollado múltiples estudios analizando los efectos de los polimorfismos de GTSs en la susceptibilidad a cáncer en general y a los cánceres relacionados con el tabaco en particular ${ }^{18}$. El fenotipo GSTM1, nulo ha centrado la atención y se ha asociado con el efecto marginal en la susceptibilidad genética de los cánceres relacionados con tabaco, incluyendo cáncer de vejiga ${ }^{21}$. Sin embargo, existen varios estudios informando acerca del papel del genotipo GSTT1 nulo y su riesgo en cáncer de vejiga y aunque disponemos de resultados, estos son contradictorios ${ }^{22,23}$.

La sulfotransferasa 1A1 (SULT1A1) y el citocromo P450 1B1 (CYP1B1) también están involucrados en el metabolismo de las PAHs y de la aminas aromáticas. SULT1Al pertenece a la superfamilia de genes que están involucrados en el metabolismo de xenobióticos vía sulfonación ${ }^{24}$. La sulfonación es un paso común en la detoxificación, sin embargo, los aniones sulfatados pueden ser descompuestos (limpiados) de manera heterotípica y liberar sus electrofilicos que pueden unirse al $\mathrm{ADN}^{25}$. El cambio de un nucleótido, de guanosina (G) a adenina (A) que conlleva a un cambio de aminoácidos, de arginina a histidina en el codon 213, ha sido identificado y puede conllevar una menor actividad enzimática y termoestabilidad $^{26}$. CYP1B1 activa carcinógenos relacionados con el tabaco como dihidrodioles$\mathrm{PAH}^{27}$. Un polimorfismo identificado ha sido el cambio de un nucleótido, de guanosina (G) a adenina (A), que conlleva un cambio de aminoácidos, de valina a leucina en el codón $432^{28}$. Con el alelo leucina 432, que posee una menor eficiencia catalítica para la 4-hidroxilación que el fenotipo salvaje $^{29}$, sugiere una posible reducción del riesgo de cáncer.

Se ha hipotetizado sobre el efecto que las variaciones genéticas de estos genes metabólicos tienen en la susceptibilidad individual al cáncer de vejiga, demostrándose un mayor riesgo de pa- decer cáncer de vejiga en los genotipos GSTT1 nulo y GSTM1 nulo, con una odds ratio de 1,69 $(\mathrm{IC} 95 \%=1,1-2,56)$ y de $1,69(\mathrm{IC} 95 \%=1,02-2,95)$ respectivamente. El efecto de GSTM1 nulo se ha visto particularmente en grandes fumadores, existiendo un efecto combinado con la exposición a aminas aromáticas (OR=2,77 IC95\%=1,08-7,10). Asímismo se observó una tendencia aumentada de riego de cáncer comparando sujetos con genotipo GSTT1 y GSTM1 normal comparándolos con aquellos que presentasen uno o dos genotipos nulos. El polimorfismo de SULT1A1 (Arg 213 His) ha demostrado $\mathrm{u}$ efecto protector en la génesis del cáncer de vejiga ${ }^{18}$. Resultados que sugieren que la susceptibilidad individual al cáncer de vejiga está modulada por los polimorfismos de GSTT1, GSTM1 y SULT1A1.

De esta manera, los objetivos que nos hemos planteado son los siguientes:

1. Analizar la expresión del factor de trascripción SXR y del MDRl en vejiga mediante RT-PCR en tiempo real, tanto en vejiga tumoral como vejiga normal.

2. Analizar la relación de los factores clínicos y patológicos con la expresión del SXR y del MDR1.

3. Analizar la expresión de los polimorfismos de CYP1B1, GSTM1 GSTT1 y SULT1A1, y su correlación con distintos factores clínico patológicos y moleculares.

\section{MATERIAL Y MÉTODOS}

En 67 pacientes de dos instituciones distintas (Hospital Universitario Miguel Servet (49 HUMS) y Clínica Universitaria de Navarra (18 CUN)), diagnosticados de cancer vesical infiltrante y tratados mediante cistectomía radical, se le realizó la determinación de la expresión de SXR y MDR1 así como de los polimorfismos CYP1B1, GSTM1 GSTT1 y SULT1A1. El tamaño de la muestra se había calculado previamente, en el momento del planteamiento de la hipótesis, considerando para el cálculo de la muestra, que la tasa de respuesta en cáncer de vejiga avanzado se cifra en un $30 \%(\varepsilon=0,30)$. De acuerdo con las tablas de Machin et $\mathrm{al}^{30}$ con $\alpha=0,05$ unilateral y una potencia de contraste del $80 \%(b=0,20)$ unilateral, el número necesario de pacientes evaluables es de 72 pacientes (IC 95\%: 61-79). Considerando un porcentaje de pacientes no evaluables del $5 \%$, el 
total de pacientes a reclutar era de 75 (IC 95\%: 64-81). Este tamaño muestral está incluido dentro del intervalo de confianza al 95\% del cálculo muestral.

La edad media de los pacientes al diagnóstico era de 64,4 años (rango $47-88$ años). 57 pacientes $(85,1 \%)$ eran hombres y $10(14,9 \%)$ eran mujeres.

La edad media de los pacientes al diagnóstico era de 64,4 años (rango 47 - 88 años). 57 pacientes $(85,1 \%)$ eran hombres y $10(14,9 \%)$ eran mujeres.

Con el diagnóstico anatomopatológico (RTU de vejiga) de tumor vesical infiltrante. Todos ellos fueron sometidos a cistectomía radical con derivación urinaria, bien ortotópica (Hautmann, Camey II) o heterotópica (Briker Wallace II, Mainz II), con linfadenectomía ilio-obturatriz ampliada.

En el momento de la cirugía se extrajeron muestras de vejiga tumoral y no tumoral determinado por un especialista en anatomía patológica, determinando la presencia de más de un $75 \%$ de células tumorales en la muestra. Las muestras se congelaron a $-80^{\circ} \mathrm{C}$ hasta la extracción de ácidos nucleicos.

Asímismo, previo consentimiento informado del paciente y antes de la cirugía se extrajeron de $20 \mathrm{ml}$ de sangre para la determinación de los polimorfismos.

Las variables patológicas obtenidas tras el análisis histopatológico de la pieza quirúrgica quedan reflejadas en la Tabla 1.

Análisis de la expresión génica mediante PCR cuantitativa en tiempo real

La expresión génica de SXR y MDR1 fue determinada en tejido tumoral y normal de las muestras de 67 pacientes.

La extracción de ARN total se llevó a cabo mediante el reactivo Trizol, total RNA isolation reagent (Gibco BRL, Life Technologies, Gaitherburg, MD, USA) de acuerdo con las instrucciones del fabricante. Todo el ARN utilizado en este estudio fue lavado utilizando el kit RNeasy Mini Kit (Quiagen, Valencia, CA) y fue tratado con exhaustivamente RNase-free DNAse I (Quiagen) para remover el AND residual.

$2 \mu \mathrm{g}$ de ARN total de cada muestral se utilizaron para generar ADNc utilizando el kit de Trans-
Tabla 1. Variables patológicas

\begin{tabular}{|c|c|c|}
\hline Variable & Número & Porcentaje \\
\hline \multicolumn{3}{|l|}{ Grado } \\
\hline II & 11 & 16,4 \\
\hline III & 56 & 81,6 \\
\hline \multicolumn{3}{|c|}{ Infiltración vasculo-linfática } \\
\hline Sí & 19 & 28,3 \\
\hline No & 48 & 71,7 \\
\hline \multicolumn{3}{|l|}{ Margen Quirúrgico } \\
\hline No & 65 & 97 \\
\hline $\mathrm{Si}$ & 2 & 3 \\
\hline \multicolumn{3}{|l|}{ Ca in situ } \\
\hline No & 34 & 50,7 \\
\hline $\mathrm{Si}$ & 33 & 49,3 \\
\hline \multicolumn{3}{|l|}{ Estadio Patológico } \\
\hline pTO & 6 & 10 \\
\hline pT1 & 8 & 16 \\
\hline pT2a & 6 & 8 \\
\hline pT2b & 13 & 20 \\
\hline pT3a & 15 & 26 \\
\hline pT3b & 13 & 10 \\
\hline pT4 & 7 & 10 \\
\hline No & 51 & 75,5 \\
\hline N1 & 8 & 14,3 \\
\hline N2 & 8 & 10,2 \\
\hline \multicolumn{3}{|l|}{ Otra Neoplasia } \\
\hline No & 50 & 77,6 \\
\hline Próstata Gleason 6 & 15 & 18,4 \\
\hline Próstata Gleason $>6$ & 1 & 2 \\
\hline Otra Neoplasia & 1 & 2 \\
\hline
\end{tabular}

criptasa inversa Taqman Reverse Transcription Reagent kit (PE Applied Biosystems, Foster City, CA). La mezcla de la reacción se incubó a $42^{\circ} \mathrm{C}$ durante 30 minutos, seguido de una incubación a $72^{\circ} \mathrm{C}$ durante 10 minutos.

Cada ejemplar de ADNc fué analizado por triplicado utilizando ABI PRISM 7700 Sequence Detector (PE Applied Biosystems) PCR- Tiempo real con nucleasa 5' "Taqman" para la detección deSXR y MDR1 se llevó a cabo utilizando Universal PCR Master Mix (Applied Biosystems), que contiene ROX para normalizar las emisiones. Los primers y las sondas se adquirieron a Applied Biosystems como "Assay on demand" (Hs00243666_ml and Hs001845000_ml). Para los ciclos térmicos, se aplicaron las siguientes condiciones: $10 \mathrm{~min}$. A $95^{\circ} \mathrm{C}$ y 40 ciclos de 15 segundos a $95^{\circ} \mathrm{C}$ y $1 \mathrm{~min}$. a $59^{\circ} \mathrm{C}$.

La Beta-actina se utilizó como control endógeno y los datos obtenidos se representaron según la fórmula $2^{-\Delta \Delta C T}$. La cuantificación relativa de la expresión de cada gen se calculó de 
acuerdo al método de comparación de ciclo límite (Ct) utilizando la $\beta$-actina como control endógeno (Hs999999903_m1) y la mediana de todas las muestras de normales como calibrador. El

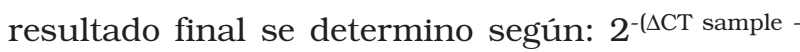
$\Delta \mathrm{CT}$ calibrator) donde el valor del $\Delta \mathrm{CT}$ del calibrador y de las muestras fueron calculados mediante la resta del valor del Ct diana, del valor de la $\beta$-acti$\mathrm{na}^{31}$. El valor del Ct se determina como el ciclo en el cual la fluorescencia emitida es significativamente mayor que los niveles de fondo. Se aceptó, en todos los experimentos, una desviación estándar del valor del $\mathrm{Ct}<0,35$.

\section{Realización de los polimorfismos}

Hemos realizado el análisis de los siguientes polimorfismos en pacientes diagnosticados de tumor vesical infiltrante y tratados mediante cistectomía. La técnica elegida para el análisis de los polimorfismos del estudio ha sido la técnica RFLP (Restriction fragment length polymorphisms). Esta técnica conjuga la amplificación mediante PCR de la región genómica que incluye el polimorfismo de interés con la utilización de enzimas de restricción cuyo punto de corte en la secuencia evidencia un patrón de bandas distinto en los sujetos portadores del polimorfismo analizado.

Con esta técnica, se estudiaron los polimorfismos de los genes CYP1B1, GSTT1, GSTM1 y SULT1A1, mediante amplificación de las regiones que tuvieran en su secuencia el polimorfismo buscado, hasta alcanzar cantidades suficientes de producto con el fin de que fueran detectadas mediante electroforesis en gel.

Las reacciones de PCR se llevaron a cabo en un volumen total de $50 \mu \mathrm{l}$, preparando previamente una mezcla de todos los reactivos y añadiendo posteriormente 100 ngr de ADN. Los reactivos utilizados para la amplificación génica se adquirieron en la casa comercial Applied Biosystems (USA).

\section{Tratamiento $y$ seguimiento}

$\mathrm{El}$ tratamiento fue cirugía exclusivamente en 50 pacientes $(74,6 \%) ; 13$ pacientes $(19,4 \%)$ fueron sometidos a terapia adyuvante con quimioterapia según esquema del hospital donde se trátese el paciente, 4 pacientes (6\%) fueron sometidos a quimioterapia y radioterapia adyuvante con una dosis de 45 Gy.
Las indicaciones de adyuvancia fueron de acuerdo a los criterios de las dos instituciones. En el HUMS las indicaciones fueron en coincidencia con la recomendación de las guías de la $\mathrm{EAU}^{32}$, siempre que la toxicidad previsible fuera asumible médica y personalmente por el paciente.

El protocolo de seguimiento se realizó en base a las guías clínicas de la Asociación Europea de Urología (EAU) ${ }^{32}$ y a las guías clínicas de la $\mathrm{NCCN}^{33}$.

\section{Análisis estadístico}

Para el análisis estadístico se utilizó el software SSPP 13.0®.

Para comparación de variables cuantitativas dentro del mismo paciente se ha realizado comparación de medias mediante la prueba $\mathrm{T}$ para muestras relacionadas.

Para comparación de variables cuantitativas se ha realizado comparación de medias mediante t-Student en caso de distribución normal. Se ha realizado un test de Mann Whitney en caso de distribución no normal.

Para variables cualitativas se han realizado tablas de de contingencia $2 \times 2$ según $F$ exacta de Fisher, o 2xb según Chi cuadrado.

\section{RESULTADOS}

\section{Estudio descriptivo}

La media de seguimiento de los pacientes fue de 23,7 meses, con una mediana de 28,26 meses, con un rango de 2 meses a 80 meses.

De los 67 pacientes estudiados, 31 pacientes $(46,3 \%)$ presentaron progresión de la enfermedad, bien en forma de recidiva local, metástasis a distancia o ambos, con un tiempo medio a recidiva de 12,4 meses, mediana de 10 meses, con un rango de 1,1 mes a 31,9 meses. 36 pacientes $(53,7 \%)$ no presentaron evidencia de progresión de la enfermedad.

El estado actual de los pacientes es el siguiente: 35 pacientes $(52,2 \%)$ se encuentran vivos y libres de progresión; 8 pacientes $(11,9 \%)$ se encuentran en progresión; 21 pacientes $(31,3 \%)$ han muerto como consecuencia de la enfermedad; 3 pacientes $(4,5 \%)$ han muerto por otras causas.

Estudio de la expresión de SXR y MDR1 en vejiga

Tanto el receptor de esteroides y xenobióticos SXR como el Multidrug resistance gene (MDR1), 
se expresan en vejiga normal y en vejiga tumoral. de la pieza de cistectomía. Hemos analizado su expresión de manera cuantitativa y de manera cualitativa. Realizando en cada par (tumor y normal) $\log 2$ (ratio) con el fin de determinar la sobreexpresión o no del gen entre el tumor y su propio tejido normal, con el objetivo de calificar la expresión de una manera cualitativa. Para ello se han clasificado en infra-regulados (down-regulated) $(<-0.8)$, no cambios $(-0.8+0.8)$ y sobre-regulados (up-regulated) (>+0.8). También se ha creado otra variable en las que se distingue sobre-expresados versus no sobre-expresados. (Tabla 2).

$\mathrm{Su}$ expresión, tanto en vejiga normal, de la pieza de cistectomía, como vejiga tumoral, no sigue una distribución normal (Fig. 1).

Tabla 2. Resultados de la expresión de SXR y MDR1

\begin{tabular}{lcc}
\hline Expresión & SXR & MDR1 \\
\hline $\begin{array}{l}\text { Cuantitativa } \\
\quad \text { Vejiga tumoral (mediana) }\end{array}$ & $0,94 \Delta \mathrm{Ct}$ & $0,94 \Delta \mathrm{Ct}$ \\
$\begin{array}{l}\text { Cuantitativa } \\
\quad \text { Vejiga tumoral (mediana) }\end{array}$ & $1,09 \Delta \mathrm{Ct}$ & $0,45 \Delta \mathrm{Ct}$ \\
$\begin{array}{l}\text { Cualitativa } \\
\quad \text { Infrarregulado }\end{array}$ & $21(31,3 \%)$ & $27(40,3 \%)$ \\
$\quad$ No Cambios & $21(31,3 \%)$ & $31(46,3 \%)$ \\
$\quad$ Sobre-regulados & $25(37,3 \%)$ & $9(13,4 \%)$ \\
$\begin{array}{l}\text { Cualitativa } \\
\quad \text { No Sobre expresión }\end{array}$ & $21(31,3 \%)$ & $58(86,6 \%)$ \\
$\quad$ Sobre expresión & $46(68,7 \%)$ & $9(13,4 \%)$ \\
\hline
\end{tabular}

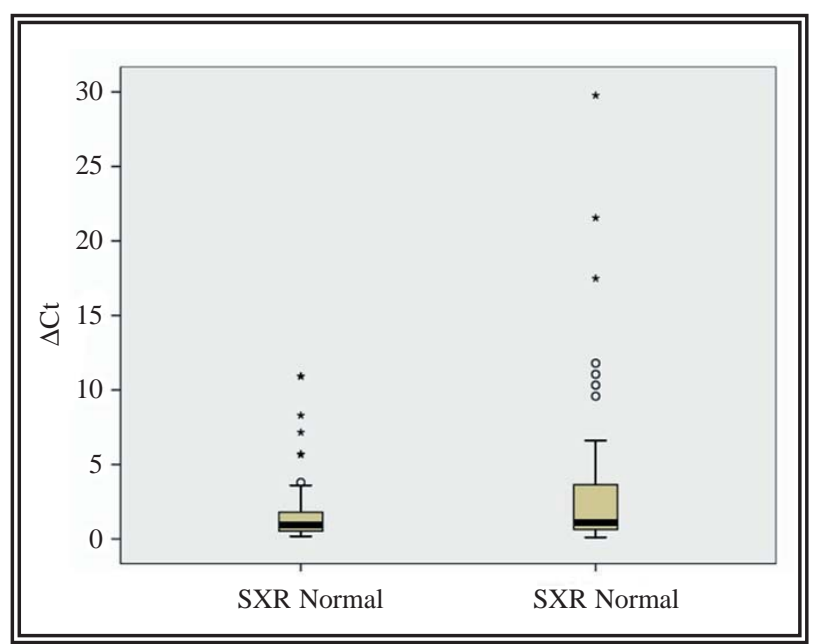

FIGURA 1. Expresión de SXR tanto en vejiga normal como tumoral, se observa que su expresión en ambos casos, no sigue una distribución normal.
Analizando si existe una correlación entre la expresión de SXR en vejiga normal y tumoral, ambas de manera cuantitativa, esta no es estadísticamente significativa ( $\mathrm{p}=0,718)$.

Sus expresiones, tanto en vejiga normal, de la pieza de cistectomía, como tumoral, no siguen una distribución normal. Su expresión es significativamente menor en el tumor que en el tejido normal ( $\mathrm{p}=0,001$ ) (Fig. 2).

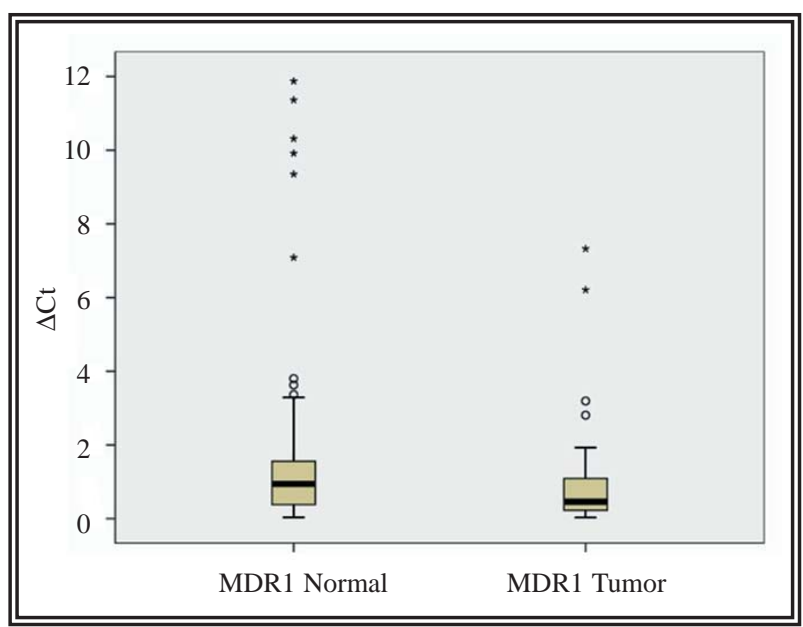

FIGURA 2. Curvas de expresión de MDR1 en vejiga normal y tumoral, se observa que la expresión en ambos casos, no sigue una distribución normal.

Analizando si existe una correlación entre la expresión de MDRl en vejiga normal y tumoral, ambas de manera cuantitativa, esta es estadísticamente significativa $(\mathrm{p}<0,0001)$.

\section{Estudio descriptivo de los polimorfismos}

Se han analizado la expresión de los siguientes polimorfismos: CYP1B1, GSTM1, GSTT1 y SULT1A1, que han seguido la siguiente distribución (Tabla 3).

Correlato de los factores clínicos y patológicos con la expresión de SXR y MDR1

La expresión de SXR en vejiga normal, no guarda correlación con ninguno de los factores pronósticos clínicos estudiados (hidronefrosis, fumador) ni con ninguno de los factores pronósticos patológicos estudiados, como la presencia de carcinoma in situ, estadio patológico, infiltración vasculo-linfática, grado histológico de diferenciación, márgenes quirúrgicos. Ni con el estado del paciente. 
Tabla 3. Estudio descriptivo

\begin{tabular}{lcc}
\hline & N & Porcentaje \\
\hline CYP1B1 & & \\
GG & 21 & 31,1 \\
GC & 27 & 41 \\
CC & 19 & 27,9 \\
Total & 67 & 100 \\
GSTM1 & & \\
Presente & 25 & 36,1 \\
Nulo & 42 & 63,9 \\
Total & 67 & 100 \\
GSTT1 & & \\
Presente & 55 & 85,2 \\
Nulo & 12 & 14,8 \\
Total & 67 & 100 \\
SULT1A1 & & \\
GG & 35 & 54 \\
GA & 26 & 39,3 \\
AA & 6 & 6,6 \\
Total & 67 & 100 \\
\hline
\end{tabular}

Analizando la expresión cuantitativa de SXR en vejiga tumoral, observamos que existe una correlación positiva con la presencia de Carcinoma in situ $(\mathrm{p}=0,014)$ así como con el estado actual de los pacientes, agrupado en vivo libre de enfermedad, o en progresión o muerte por enfermedad. $(p=0,015)$ (Tabla 4). No existe correlación estadísticamente significativa con ninguna de las restantes variables.

Tabla 4. Correlación de la expresión cuantitativa de SXR con Ca. in situ y estado actual

\begin{tabular}{lcccc}
\hline & $\mathbf{n}$ & $\begin{array}{c}\text { SXR vejiga } \\
\text { tumoral }(\Delta \mathbf{C t})\end{array}$ & IC 95\% & Valor p \\
\hline Ca in situ & & & & \\
No & 34 & 2,18 & $1,19-3,18$ & \\
$\mathrm{Si}$ & 33 & 4,68 & $2,22-7,15$ & 0,014 \\
$\begin{array}{l}\text { Estado } \\
\text { Vivo }\end{array}$ & 35 & 1,88 & $1,02-2,79$ & \\
$\begin{array}{l}\text { Progresión } \\
\text { o Muerte }\end{array}$ & 32 & 5,4 & $2,64-8,16$ & 0,015 \\
\hline
\end{tabular}

Analizando la expresión cualitativa del SXR en vejiga tumoral y observando su correlación con el resto de factores pronósticos (tablas 2x2), observamos que existe una correlación estadísticamente significativa con la presencia de carcinoma in situ $(\mathrm{p}=0,024)$, con el estado de los pacientes $(\mathrm{p}=0,01)$, con la infiltración vasculo linfática $(\mathrm{p}=0,05)$ y con la expresión del MDR1 ( $\mathrm{p}=0,011)$. No observando ninguna relación con el estadio patológico $(\mathrm{p}=0,115) \mathrm{ni}$ con el grado histológico $(\mathrm{p}=0,478)$ (Tabla 5).
Tabla 5. Correlación expresión cualitativa SXR con Ca in situ infiltración vasculo-linfática y MDR1

\begin{tabular}{lccc}
\hline SXR & $\begin{array}{c}\text { No sobre- } \\
\text { expresión } \\
\text { N }\end{array}$ & $\begin{array}{c}\text { Sí Sobre- } \\
\text { expresión } \\
\mathbf{N}\end{array}$ & Valor p \\
\hline Ca in situ No & 26 & 8 & \\
Ca in situ Si & 16 & 17 & 0,024 \\
$\begin{array}{l}\text { Infiltración } \\
\text { Vasc-Linf No }\end{array}$ & 33 & 14 & \\
$\begin{array}{l}\text { Infiltración } \\
\text { Vasc-Linf Sí }\end{array}$ & 8 & 11 & 0,05 \\
$\begin{array}{l}\text { MDR1 No sobre } \\
\text { expresado }\end{array}$ & 40 & 18 & \\
$\begin{array}{l}\text { MDR1 Sobre } \\
\text { expresado }\end{array}$ & 2 & 7 & 0,011 \\
\hline
\end{tabular}

Analizando la expresión cuantitativa de MDR1 en vejiga tumoral, observamos que existe una correlación positiva con la presencia de hidronefrosis $(p=0,024)$ (Tabla 6). No existe correlación estadísticamente significativa con ninguna de las restantes variables, tanto clínicas como patológicas.

Tabla 6. Correlación entre la expresión cuantitativa de MDR1 en tumor con hidronefrosis.

\begin{tabular}{lccc}
\hline Hidronefrosis & N & $\begin{array}{c}\text { MDR1 Vejiga } \\
\text { Tumoral }(\Delta \mathbf{C t})\end{array}$ & Valor p \\
\hline No & 51 & 37,01 & \\
$\mathrm{Si}$ & 16 & 24,01 & 0,024 \\
\hline
\end{tabular}

Analizando la expresión cuantitativa del MDR1 en vejiga tumoral (tablas $2 \times 2$ ) y observando su correlación con el resto de factores pronósticos, observamos que existe una correlación estadísticamente significativa con la presencia de infiltración vasculo-linfática $(p=0,05)$ y con la expresión de SXR de manera cualitativa $(\mathrm{p}=0,001)$ (Tabla 7$)$, la expresión de MDR1 en vejiga tumoral, de manera cualitativa no tiene ninguna correlación con el estadio patológico $(\mathrm{p}=0,921)$, ni con el grado histológico $(\mathrm{p}=0,875)$.

Correlación con Factores Pronósticos, Clínicos y Patológicos

Los polimorfismos estudiados no se correlacionan de manera estadísticamente significativa con ninguna variable clínico patológica (Tabla 8). 
Tabla 7. Correlación expresión cualitativa de MDR1

\begin{tabular}{lcccc}
\hline MDR1 & $\begin{array}{c}\text { Infra } \\
\text { Regulado } \\
\mathbf{N}\end{array}$ & $\begin{array}{c}\text { No } \\
\text { Cambios } \\
\mathbf{N}\end{array}$ & $\begin{array}{c}\text { Sobre } \\
\text { Regulado } \\
\mathbf{N}\end{array}$ & $\begin{array}{c}\text { Valor } \\
\mathbf{P}\end{array}$ \\
\hline $\begin{array}{l}\text { Infiltración } \\
\text { Vasc-Linf NO }\end{array}$ & 17 & 26 & 4 & \\
$\begin{array}{l}\text { Infiltración } \\
\text { Vasc-Linf SI }\end{array}$ & 9 & 5 & 5 & 0,05 \\
$\begin{array}{l}\text { SXR No } \\
\text { sobre-expresión }\end{array}$ & 20 & 20 & 2 & \\
$\begin{array}{l}\text { SXR Sí } \\
\text { sobre-expresión }\end{array}$ & 7 & 11 & 7 & 0,02 \\
\hline
\end{tabular}

Tabla 8. Correlación con factores pronósticos

\begin{tabular}{lcccc}
\hline & CYP1B1 & GSTM1 & GSTT1 & SULT1A1 \\
\hline Ca. in situ & 0,698 & 0,843 & 0,238 & 0,334 \\
pTNM & 0,811 & 0,395 & 1 & 0,114 \\
cTNM & 0,508 & 0,732 & 0,0521 & 0,802 \\
Estado & 0,666 & 0,649 & 0,341 & 0,787 \\
Ganglios cx & 0,291 & 0,373 & 1 & 0,201 \\
Ganglios Pat & 0,608 & 0,815 & 0,325 & 0,377 \\
Grado Histolo & 0,276 & 0,802 & 1 & 0,635 \\
GSTM1 & 0,263 & & 0,425 & 0,525 \\
GSTT1 & 0,754 & 0,425 & & 0,916 \\
Hidronefrosis & 0,317 & 0,513 & 0,771 & 0,354 \\
Infiltración VL & 0,718 & 0,543 & 1 & 0,546 \\
Margen & 0,99 & 0,566 & 1 & 0,075 \\
MDR1 & 0,696 & 0,417 & 0,660 & 0,469 \\
\hline
\end{tabular}

\section{DISCUSIŌN}

Analizando los objetivos primarios, observamos que el SXR y MDR1 se expresan en los tumores vesicales infiltrantes de vejiga, tanto en el tumor como en la vejiga normal. Este estudio supone el primer trabajo en el que se describe la expresión de SXR en tumores de vejiga. Habiéndose comunicado previamente ${ }^{34}$ la expresión de la misma por nuestro grupo de trabajo.

La expresión del MDR1 en vejiga se ha demostrado, así como su inducción por agentes quimioterápicos, poniendo en evidencia que la sobreexpresión del MDR1 puede contribuir a la resistencia a agentes citotóxicos ${ }^{16}$, pero no se ha demostrado que su expresión se asocie a un peor pronóstico.
Analizando la expresión de SXR en vejiga, tanto normal como tumoral, y analizando la correlación de la misma con los factores clínico-patológicos conocidos, observamos que la expresión del SXR en vejiga normal no guarda ninguna correlación con los factores clínico-patológicos descritos en la literatura. Sin embargo, la expresión de SXR en la vejiga tumoral, analizada de manera cualitativa, guarda una correlación estadísticamente significativa con los siguientes factores patológicos conocidos, presencia de carcinoma in situ $(p=0,0024)$, infiltración vasculo-linfática $(\mathrm{p}=0,05)$ y la expresión de MDR1 $(\mathrm{p}=0,011)$. Por otra parte, si su expresión es analizada de manera cuantitativa, observamos que, continua guardando relación con la presencia de carcinoma in situ $(\mathrm{p}=0,014)$, y se correlaciona con el estado actual de los pacientes ( $\mathrm{p}=0,015)$.

A su vez, analizando la expresión del MDR1 en vejiga normal y su correlación con los mismos factores clínico-patológicos, vemos que se correlaciona con el estado actual de los pacientes $(\mathrm{p}=0,031)$, la presencia de hidronefrosis en el momento del diagnóstico $(\mathrm{p}=0,018)$, así como la expresión de SXR en vejiga tumoral $(\mathrm{p}=0,021)$. Sin embargo, si analizamos la expresión de MDR1 en vejiga tumoral de manera cuantitativa, vemos que su expresión se correlaciona únicamente con la presencia de hidronefrosis $(\mathrm{p}=0,029)$, y si su expresión se analiza de manera cualitativa esta se correlaciona con la infiltración vasculo-linfática y la expresión de SXR en vejiga tumoral $(\mathrm{p}=0,02)$.

Estos resultados suponen, al vez que la descripción del SXR por primera vez, la correlación del mismo con factores patológicos de mal pronósticos conocidos, como son el carcinoma in situ, la infiltración vasculo-linfática y el estado actual de los pacientes. Asímismo, un dato previamente conocido en la literatura ${ }^{35}$, y que se confirma con estos resultados, es la correlación de la expresión de SXR con la de MDR1, resultado que confirma la interrelación de ambos en el metabolismo regulador de xenobióticos.

A continuación, analizaremos los resultados obtenidos del análisis de la expresión de los polimorfismos CYP1B1, GSTM1, GSTT1, y SULT1A1.

El cáncer de vejiga se ha asociado al hábito tabáquico en múltiples estudios epidemiológicos. 
Un importante número de enzimas detoxificantes son las GSTs, El subtipo mu de la GSTs ha sido descrito como detoxificador de los cancerígenos del tabaco, como las $\mathrm{PAH}$ y las aminas aromáticas. La ausencia de las clases mu y teta de las GSTs se han asociado a delecciones de los genes GSTT1 y GSTM1 ${ }^{18}$, la frecuencia de los genotipos GSTM1 nulo y GSTM1 nulo varía con la raza étnica y con la nacionalidad (Tablas $9 \mathrm{y}$ 10), nuestros resultados de frecuencias son similares a los obtenidos en otras serie con similares características socio-geográficas.

Respecto a la significación que pueden tener los polimorfismos y su influencia en cuanto al riesgo de padecer cáncer de vejiga, así como su influencia en el pronóstico, es un tema de controversia en la literatura. En nuestro caso ninguno de estos polimorfismos se asocia con ninguna variable de influencia en la supervivencia, ni con el hábito tabáquico, no pudiéndose asociar a un mayor riesgo de desarrollar cáncer de vejiga por no disponer de un grupo control con quien realizar la comparación

\section{CONCLUSIONES}

Analizando los resultados del presente trabajo, podemos concluir que el Receptor de Esteroides y Xenobióticos (SXR) y el gen de multirresistencia drogas (MDR1) se expresan tanto en vejiga normal y tumoral. Y que dicha expresión guarda una correlación con factores pronósticos de influencia en la supervivencia descritos en la literatura. Así como la existencia de una correlación entre las variables patológicas y los dos factores estudiados.

El valor de los polimorfismo estudiados, se expresan en vejiga con frecuencias similares a la literatura pero su expresión no guarda relación con ninguna variable estudiada.

Este trabajo supone la apertura de una linea de investigación en la que continuaremos trabajando para analizar su influencia en la supervivencia y en la relación con la resistencia a tratamientos complementarios
Tabla 9. Polimorfismos GSTM1 y GSTT1

\begin{tabular}{lccc} 
Tabla 10. Polimorfismos CYP1B1 y SULT1A1 & \\
\hline Enzima & Polimorfismo & Hung $^{\mathbf{1 8}}$ & Serie \\
\hline CYP1B1 & GG & $22 \%$ & $34,6 \%(36)$ \\
& GC & $42 \%$ & $35,6 \%(37)$ \\
SULT1A1 & GC & $36 \%$ & $29,8 \%(31)$ \\
& GG & $56 \%$ & $61 \%(64)$ \\
& GA & $38 \%$ & $33 \%(35)$ \\
& AA & $6 \%$ & $6 \%(6)$ \\
\hline
\end{tabular}

Anexo: Trabajo de investigación financiado con una Beca de la F.I.U. 2004 "Martín de Castellanos"

\section{REFERENCIAS}

1. Messing E. Urothelial Tumors of the Urinary Tract. In: Campbells Urology, 8 ed. Edited by P. Walsh, A. Retik, T. Stamey et al. Phildelphia PA.: W.B. Saunders Company, vol. 4, pp. 2732-2785, 2002.

2. Collado Serra A, Rubio Briones J, Ricos Torrent J. Generalidades sobre los tumores vesicales. In: Tratado de Urología. Edited by J. Jiménez Cruz and L. Rioja Sanz. Barcelona: Prous Science S.A., vol. IV, pp. 1851-1881, 2006.

3. Jemal A, Murray T, Ward E, Samuels A, Tiwari RC, Ghafoor A, et al. Cancer statistics, 2005. Cancer J Clin. 2005;55(1): 10-30.

4. Instituto Nacional de Mortalidad por cáncer de Vejiga: INE, 2007.

5. Herr H, Jakse G, Sheinfeld J. El tumor vesical T1. In: Casos prácticos de Urología. Edited by K. Brasilis and M. Soloway: Ed. Ep, vol. 3, pp. 1-11, 1995.

6. Blumberg B, Kang H, Bolado J Jr, Chen H, Craig AG, Moreno TA, et al. BXR, an embryonic orphan nuclear receptor activated by a novel class of endogenous benzoate metabolites. Genes Dev. 1998;12(9):1269-1277.

7. Geick A, Eichelbaum M, Burk O. Nuclear receptor response elements mediate induction of intestinal MDR1 by rifampin. J Biol Chem. 2001;276(18):14581-14587.

8. Lehmann JM, McKee DD, Watson MA, Willson TM, Moore JT, Kliewer SA. The human orphan nuclear receptor PXR is activated by compounds that regulate CYP3A4 gene expression and cause drug interactions. J Clin Invest. 1998;102(5):1016-1998.

9. Bertilsson G, Heidrich J, Svensson K, Asman M, Jendeberg L, Sydow-Bäckman M, et al. Identification of a human nuclear receptor defines a new signaling pathway for CYP3A induction. Proc Natl Acad Sci U S A. 1998;95(21): 12208-12213.

10. Miki Y, Suzuki T, Tazawa C, Blumberg B, Sasano H. Steroid and xenobiotic receptor (SXR), cytochrome P450 3A4 and multidrug resistance gene 1 in human adult and fetal tissues. Mol Cell Endocrinol. 2005;231(1-2): 75-85. 
11. Willson TM, Kliewer SA. PXR, CAR and drug metabolism Nat Rev Drug Discov. 2002;1(4): 259-266.

12. Synold TW, Dussault I, Forman BM. The orphan nuclear receptor SXR coordinately regulates drug metabolism and efflux. Nat Med. 2001;7(5):584-590.

13. Xie W, Evans RM. Orphan nuclear receptors: the exotics of xenobiotics. J Biol Chem. 2001;276(41):37739-37742.

14. Dussault I, Forman BM. The nuclear receptor PXR: a master regulator of "homeland" defense. Crit Rev Eukaryot Gene Expr. 2002;12(1):53-64.

15. Kliewer SA, Goodwin B, Willson TM. The nuclear pregnane $\mathrm{X}$ receptor: a key regulator of xenobiotic metabolism. Endocr Rev. 2002;23(5):687-702.

16. Nezasa S, Fujihiro S, Deguchi T, Kawada Y, Kawamoto S, Tamaki M, et al. Analysis of induction of MDR1 gene expression by anticancer chemotherapy in bladder cancer. Hinyokika Kiyo. 1997;43(9):629-636.

17. Kogenvians M, Trichopoulus D. Urinary bladder cancer. In: Textbook of cancer epiemiology. Edited by H. Addami, D. Hunter, D. Trichopoulus. New York: Oxford University Press. 2002;446-466.

18. Hung RJ, Boffetta P, Brennan P, Malaveille C, Hautefeuille A, Donato F, et al. GST, NAT, SULT1A1, CYP1B1 genetic polymorphisms, interactions with environmental exposures and bladder cancer risk in a high-risk population. Int J Cancer. 2004; 110(4):598-604.

19. Strange R, Fryer A. The glutathione S-transferases: influence of polymorphism on cancer susceptibility. IARC Sci Publ. 1999;(148):231-249.

20. Landi S. Mammalian class theta GST and differential susceptibility to carcinogens: a review. Mutant Res. 2000;463 (3):247-283.

21. Engel LS, Taioli E, Pfeiffer R, Garcia-Closas M, Marcus PM, Lan Q, et al. Pooled analysis and meta-analysis of glutathione S-transferase M1 and bladder cancer: a HuGE review. Am J Epidemiol. 2002;156(2):95-109.

22. Giannakopoulos X, Charalabopoulos K, Baltogiannis D, Chatzikiriakidou A, Alamanos Y, Georgiou I, et al. The role of N-acetyltransferase-2 and glutathione S-transferase on the risk and aggressiveness of bladder cancer. Anticancer Res. 2002;22(6B):3801-3804.

23. Lee SJ, Cho SH, Park SK, Kim SW, Park MS, Choi HY, et al. Combined effect of glutathione S-transferase M1 and T1 genotypes on bladder cancer risk. Cancer Lett. 2002;177 (2):173-179.

24. Glatt $\mathrm{H}$, Engelke $\mathrm{CE}$, Pabel $\mathrm{U}$, Teubner W, Jones $\mathrm{AL}$, Coughtrie MW, et al. Sulfotransferases: genetics and role in toxicology. Toxicol Lett. 2000;112-113:341-348.

25. Hengstler JG, Arand M, Herrero ME, Oesch F. Polymorphisms of $\mathrm{N}$-acetyltransferases, glutathione S-transferases, microsomal epoxide hydrolase and sulfotransferases: influence on cancer susceptibility. Recent Results Cancer Res. 1998;154:47-85.
26. Engelke CE, Meinl W, Boeing H, Glatt H. Association between functional genetic polymorphisms of human sulfotransferases 1A1 and 1A2. Pharmacogenetics. 2000;10(2): 163-169.

27. Shimada T, Watanabe J, Kawajiri K et al. Catalytic properties of polymorphic human cytochrome $\mathrm{P} 450$ 1B1 variants. Carcinogenesis. 1999;20(8):1607-1613

28. Bailey LR, Roodi N, Dupont WD, Parl FF. Association of cytochrome P450 1B1 (CYP1B1) polymorphism with steroid receptor status in breast cancer. Cancer Res. 1998;58 (22):5038-5041.

29. Hanna IH, Dawling S, Roodi N, Guengerich FP, Parl FF. Cytochrome P450 1B1 (CYP1B1) pharmacogenetics: association of polymorphisms with functional differences in estrogen hydroxylation activity. Cancer Res. 2000;60(13): 3440-3444.

30. Machin D, Campbell M, Fayers P, et al. Sample Size tables for clinical studies, Second ed. Oxford: Ed. Blackwell, pp. 23-29, 1997

31. Applied., Biosystem.: ABI Prism 7700 Sequence Detection System. User Bulletin, 2: 11, 2001.

32. Van der Meijden AP, Sylvester R, Oosterlinck W, Solsona E, Boehle A, Lobel B, et al. for the EAU Working Party on Non Muscle Invasive Bladder Cancer. EAU guidelines on the diagnosis and treatment of urothelial carcinoma in situ. Eur Urol. 2005;48(3):363-371.

33. Montie JE, Abrahams NA, Bahnson RR, Eisenberger MA, El-Galley R, Herr HW, et al. Bladder cancer. Clinical guidelines in oncology. J Natl Compr Canc Netw. 2006;(10): 984-1014.

34. Rioja Zuazu J, Bandrés E, Rosell Costa D, et al. Expresión del SXR en tumores vesicales infiltrantes. Abstract Congres. Actas Urol Esp. 6:45, 2005.

35. Zhou C, Tabb MM, Sadatrafiei A, Grün F, Blumberg B. Tocotrienols activate the steroid and xenobiotic receptor, SXR, and selectively regulate expression of its target genes. Drug Metab Dispos. 2004(10):1075-1082.

36. Kim H, Kim WJ, Lee H.-L, et al.: A case-control study on the effects of the genetic polymorphisms of $\mathrm{N}$-acetyltransferase 2 and glutathione S-transferase mu and theta on the risk of bladder cancer. Korean J Prev Med.1998;31: 275.

37. Katoh $\mathrm{T}$, Inatomi $\mathrm{H}$, Kim $\mathrm{H}$, Yang $\mathrm{M}$, Matsumoto $\mathrm{T}$, Kawamoto T. Effects of glutathione S-transferase (GST) M1 and GSTT1 genotypes on urothelial cancer risk. . Cancer Lett. 1998;132(1-2):147-152.

Correspondencia autor: Dr. J. Rioja Zuazu

Dept. Urología Clínica Universitaria. Universidad de Navarra

Av. Pio XII 36, Pamplona 31080. Tel.: 948255400

E-mail autor: jriojazu@gmail.com

Información artículo: Original - Cáncer de vejiga. Investigación

Trabajo recibido: abril 2007

Trabajo aceptado: junio 2007 